\title{
Distributed Systems and Recent Innovations: Challenges Benefits and Security Issues in Distributed Systems.
}

\author{
S.G. Bhagwath and Dr. Mallikarjun Math
}

\begin{abstract}
From the last thirty years the trends within the Computing trade is towards Distributed, low price unit, and high unit volume product. additional computer code development activity is changing into a lot of decentralized thereby increasing more development efforts for organizations. The performance of system has enhanced drastically with the inclusion of data processing and Multi computing. This paper presents a scientific literature review on the problems, Recent Innovations, Challenges and advantages of Distributed Systems. With AN example. This paper presents a the safety problems related to those systems. Four usually used distributed systems were thought-about for careful analysis in terms of technologies concerned, security problems sweet-faced by them and resolution projected to avoid those problems. Finally the safety problems and also the solutions were summarized and compared with one another. This paper is additionally aimed to grant a report on the Distributed Computing Technologies in activity.
\end{abstract}

Keywords--- Alchemi, Computing Clusters, Distributed Systems, Distributed Storage System Security, Grid System Security.

\section{INTRODUCTION}

$\mathrm{T}$ HE planet Wide net is employed by countless people everyday for numerous functions as well as email, reading news, downloading music, on-line shopping or just accessing information regarding something. employing a customary application program, the user will access info hold on on net servers placed any place on the world. This offers the illusion that each one this info is placed domestically on the user's pc. In reality, the net represents an enormous distributed system that seems as one resource to the user out there at the press of a button.

There square measure many definitions and examine points on what distributed systems are. Coulouris defines a distributed system as "a system within which hardware or software system parts settled at networked computers communicate and coordinate their actions only by message passing" [1]; and Tanenbaum defines it as "A assortment of freelance computers that seem to the users of the system as one computer" [2]. Leslie Lamport -

S.G. Bhagwath Research scholar, KLSGIT.

Dr. Mallikarjun Math, Professor in CSE, KLSGIT.

DOI:10.9756/BIJSESC.8239 a noted scientist on temporal arrangement, message ordering, and clock synchronization in distributed systems once aforementioned that "A distributed system is one on that I cannot get any work done as a result of some machine I even have never detected of has crashed" reflective on the large range of challenges faced by distributed system designers. Despite these challenges, the advantages of distributed systems and applications are many, creating it worthy to pursue.

Various styles of distributed systems and applications are developed and square measure getting used extensively within the universe. during this article, we tend to gift the most characteristics of distributed systems and appearance at a number of the challenges that square measure faced by designers and implementers of such systems, and additionally introduce an example distributed system..

\section{MAIN OPTIONS AND ADVANTAGES OF A DISTRIBUTED SYSTEM}

A common idea among individuals once discussing distributed systems is that it's simply another name for a network of computers. However, this overlooks a crucial distinction. A distributed system is made on prime of a network and tries to cover the existence of multiple autonomous computers. It seems as one entity providing the user with no matter services area unit needed. A network could be a medium for interconnecting entities (such as computers and devices) enabling the exchange of messages supported well-known protocols between these entities, that area unit expressly available (using Associate in Nursing IP address, for example).

There area unit numerous forms of distributed systems, Clusters [3], Grids [4], P2P (Peer-to-Peer) networks [5], distributed storage systems then on Units.

A cluster could be a dedicated cluster of interconnected computers that seems as one super-computer, typically utilized in high performance scientific engineering and business applications. A grid could be a kind of distributed system that permits coordinated sharing and aggregation of distributed, autonomous, heterogeneous resources supported users' QoS (Quality of Service) necessities. Grids area unit unremarkably wont to support applications rising within the areas of e-Science and e-Business, that unremarkable geographically distributed communities of individuals World Health Organization interact in cooperative activities to resolve massive scale issues and need sharing of as 
sorted resources adore computers, data, applications and scientific instruments. P2P networks area unit localized distributed systems, that change applications adore filesharing, instant electronic messaging, on-linemultiuser vice and content distribution over public networks. Distributed storage systems adore NFS (Network File System) offer users with a unified read of knowledge hold on on totally different file systems and computers which can get on identical or different networks..

The independent features of a distributed system include [1] [2]:

\section{A. Functional Separation}

Based on the functionality/services provided, capability and purpose of each entity in the system.

\section{B. Inherent Distribution}

Entities such as information, people, and systems are inherently distributed. For example, different information is created and maintained by different people. This information could be generated, stored, analysed and used by different systems or applications which may or may not be aware of the existence of the other entities in the system.

\section{Reliability}

Long term data preservation and backup (replication) at different locations.

\section{Scalability}

Addition of more resources to increase performance or availability.

\section{E. Economy}

Sharing of resources by many entities to help reduce the cost of ownership.

As a consequence of these features, the various entities in a distributed system can operate concurrently and possibly autonomously. Tasks are carried out independently and actions are co-ordinated at well-defined stages by exchanging messages. Also, entities are heterogenous, and failures are independent. Generally, there is no single process, or entity, that has the knowledge of the entire state of the system Various kinds of distributed systems operate today, each aimed at solving different kinds of problems. The challenges faced in building a distributed system vary depending on the requirements of the system. In general, however, most systems will need to handle the following issues [1] [2]:

\section{F. Heterogeneity}

Various entities in the system must be able to interoperate with one another, despite differences in hardware architectures, operating systems, communication protocols, programming languages, software interfaces, security models, and data formats.

\section{G. Transparency}

The entire system should appear as a single unit and the complexity and interactions between the components should be typically hidden from the end user.

\section{H. Fault Tolerance and Failure Management}

Failure of one or more components should not bring down the entire system, and should be isolated.

\section{Scalability}

The system should work efficiently with increasing number of users and addition of a resource should enhance the performance of the system.

\section{J. Concurrency}

Shared access to resources should be made possible.

\section{K. Openness and Extensibility}

Interfaces should be cleanly separated and publicly available to enable easy extensions to existing components and add new components.

\section{Migration and Load Balancing}

Allow the movement of tasks within a system without affecting the operation of users or applications, and distribute load among available resources for improving performance.

\section{Security}

Access to resources should be secured to ensure only known users are able to perform allowed operations.

\section{DiSTRIBUTED COMPUTING TECHNOLOGIES IN PATTERN}

Quit the life-span, technologies such as CORBA and DCOM assault provided the power to build disappoint a amount to abettor-based systems. Such technologies acknowledge systems to interoperate at the component difference, by proviso a software coat and protocols prowl furnish the

interoperability immediately for volume developed in different programming languages to exchange messages. Though, such technologies true scalability issues straight away commonsense to, for case, the Internet and several restrict the developer to a specific programming language. Consistent back, approaches based on Twine protocols and XML (extensible Mark up Language) have a go been trivial to reconcile oneself to interoperable distributed systems irrespective the programming language in which they are developed. Revile Rite are based on XML and make consistent a activity to have distributed systems ramble follow a Service Oriented Architecture (SOA). Marines are conjectural in an XML-based dialect (WSDL). In a akin accustom, the call and admission messages returned in such systems are formatted according to the Simple Object Access Protocol (SOAP). SOAP messages basis be cryptographic and transmitted by partake of Catch on to protocols such as the Hypertext Transfer Protocol(HTTP). Unusual doctrinaire technologiesand sue plat forms such as .NET immigrant Microsoft, J2EE detach immigrant Sunlight, Weh Sphere outlander IBM are targeted at supporting the development of applications basedon Netting Assignment . Confer to Weave Ceremony, Gr 
ating computing is selection emerging paradigm for creating wide-area distributed applications. Tatting Military talents are basis technologies range hindquarters be hand-medown in edifice discrete types of distributed systems and applications including Discordant systems. Web repair are in the evil-minded of of Jangling technologies the authentic implementations such

Globus stranger Argonne Wide Laboratory in USA and the Gridbus unfamiliar the University of Melbourne, Australia. Grid computing equilibrium from an effect/organisation to a Gigantic level. wide-ranging Grids

are particular yield the do Internet coarse, and are characterizedbya voluminous aspect,pacify of extent familiar $p$ eremptory, verifiable sophisticated fasten mechanisms, focus on single sign-on and are mostly batch-job oriented. To approve globalGrids, brace title is deviate authentic Influen ce and campus Grids are able to interoperate. deed and campus Grids consist of peremptory extent buffet an enterprise and shelter services to users incarcerated that organisation and are managed by a single administrative domain. Such Grids are with reference to careful with recur thievery from new desktops and reckon for virtualization of resources in mandate to provide better means to manage and utilize them within an enterprise. For cause, Fortune-teller $10 \mathrm{~g}$ uses a virtualization beyond to gap matter storage from the database transaction and process layer. Though, scalability and the prevent a rough out of security mechanisms are shriek as trying as they are for global Grids.

\section{ALCHEMI: ASSOCIATE EXAMPLE DISTRIBUTED SYSTEM}

Intypical company or tutorial surroundings there are severa 1 resources that are usually under-utilised for long periods of your time. A "resource" during this context means that any entity that might be accustomed fulfil any user requirement; this includes figure power (CPU), information storage, applications, and services. associate enterprise grid may be a distributed system that dynamically aggregates associated coordinates numerous resources inside associate organisation and improves employment specified there's an overall increase in productivity for the users and processes. These advantages ultimately lead to vast value savings for the business, since they'll not have to be compelled to purchase price instrumentality for the aim of running their high performance applications.

The fascinating options of associate enterprise grid system are:

1. Enabling economical and best resource usage.

2. Sharing of inter-organisational resources.

3. Secure authentication and authorization of users.

4. Security of keep information and programs.

5. Secure communication.

6. Centralised / semi-centralised management.

7. Auditing.

8. Enforcement of Quality of Service (QoS) and repair Level Agreements (SLA).

9. Interoperability of various grids (and hence: the premise on open-standards).

10. Support for transactional processes.

Alchemi [6] is associate Enterprise Grid computing framework developed by researchers at the GRIDS work, within the engineering and code Engineering Department at the University of Melbourne, Australia. It permits the user to mixture the computing power of networked machines into a virtual mainframe and develop applications to run on the Grid with no further investment and no discernible impact on users.

The main options offered by the Alchemi framework are:

1. Virtualization of figure resources across the local area network / net.

2. Ease of readying and management.

3. Object-oriented "Grid thread" programming model for grid application development.

4. File-based "Grid job" model for gridenabling heritage applications.

5. Web services interface for ability with different grid middleware.

6. Open-source.Net based, straightforward installation victimisation Wind ows installers.

Alchemi Grids follow the master-slave design, with the extra capability of connecting multiple masters in an exceedingly hierarchicor peer-to-peer fashion to supply measurability of the system. associate Alchemi grid has 3 varieties of parts particularly the Manager, the fiduciary, and therefore the User Application itself.

The Manager node is that the master / controller whose main perform is to service the user requests for work distribution. It receives a user request, authenticates the user, and distributes the work across the assorted Executors that are connected to that. The fiduciary node is that the one that really performs the computation. Alchemi uses role-based security to evidence users and authorize execution. an easy grid is formed by putting in Executors on every machine that's to be a part of the grid and linking them to a central Manager element.

\section{SECURITY IN DISTRIBUTED SYSTEMS}

Security is one amongst the
foremost necessary problems in distributed systems. once information is distributed across multiple networks or info is transferred via public networks, it becomes prone to attacks by mischievous parts. equally alternative computing resources like processors, storage devices, networks etc., can even be attacked by hackers..

\section{A. Security for Computing Clusters}

When the computing clusters area unit created on the market to the general public or networks area unit setup exploitation public resources like the web, they become subject to numerous styles of attacks. the foremost common forms of attacks on the clusters area unit computation-cycle stealing, inter-node communication snooping, and cluster service disruption [17]. thus the clusters are protected by security mechanisms that embody services like authentication, integrity check, and confidentiality. the most purpose of the safety mechanisms is to guard the system against hackers moreover on meet the 
safety necessities of the applications.

Li and Vaughn have studied the safety vulnerabilities of computing clusters exploitation exploitation graphs (egraphs). they need sculptural many attacks which will be carried on all 3 pillars of security specifically, confidentiality, integrity and convenience. They need shown that graphs is simplified supported domain data like cluster configurations, detected vulnerabilities, etc. They more state that this method may be used for certification of clusters with the assistance of a cognitive content of cluster vulnerabilities[18].

Xie and Qin have developed 2 resource allocation schemes named point and Security constraints (TAPADS) and Security-Aware and Heterogeneity-Aware Resource allocation for Parallel jobs (SHARP). These 2 schemes make sure that parallel applications dead on computing clusters meet the safety necessities whereas meeting the point of executions [17]. thus it may be seen that if these schemes guarantee principally the supply of the system as timely execution of associate degree application is a sign of the supply of the resources.

Denial of Service (DoS) attack is one amongst the common attacks on distributed systems. These attack principally target resources in such a way that the resources area unit prevented from finishing up their legitimate operations. a way that uses services and Mark off chain to mitigate the results on the DoS attack on a cluster primarily based wireless sensing element network has been bestowed in [19].

Hence it is seen that computing clusters area unit susceptible to attacks by mischievous components like hackers and round the bend because of its open nature and use of public resources like the web. intensive analysis has been dole out by many-researchers on the protection of clusters and that they have planned many ways which will be created accustomed protect the clusters from these attacks.

\section{B. Grid System Security}

Grid pc systems give many security mechanisms to guard the grid resources against attacks. Middle ware is one among the important system package within the grid infrastructure because it provides the common communication infrastructure and makes the grid services accessible to applications. Middleware conjointly permits for a regular security configuration at the service instrumentality or messaging level. Grid authentication is predicated on Public Key Infrastructure (PKI) and capable of handling differing kinds of user credentials similar to PKI, SAML, Kerberos tickets, password, etc., Delegation is one among the mandatory mechanisms in grid service delivery and is enforced victimization X.509 Proxy Certificate. Authorization to access grid resources is predicated on Virtual Organization (VO) attributes allotted to a user and managed by Virtual Organization Membership Service (VOMS). Trust management in grid systems area unit handled victimization certificates and trust relations area unit painted by a certificate chain that embody Grid
Certification Authority (CA) certificate and different in turn generated proxies [20].

Grid authentication module is one among the important parts in preventing external users from arbitrarily accessing internal grid and protective the grid system from unauthorized users. This module handles security threats from internal network, once certified grid users perform outlaw (unauthorized) operations among the grid [21].

These grid security mechanisms area unit all enforced on the majority grid systems accessible nowadays. There many grid community initiatives occurring within the space of grid middleware ability which might finally unify the grid security as one coherent security platform and theme

\section{Distributed Storage System Security}

Several active researches square measure occurring within the space of threat modeling and developing security model for safeguarding distributed storage systems. the foremost necessary resource within the distributed storage system is that the knowledge keep within the storage devices of the system. This knowledge has to be properly labelled and guarded. additionally any defendion system introduced should be backward compatible in different words; it not solely ought to protect the information keep once the safety theme is put in however additionally the information that had been there before the introduction of that theme.

Hasan et al., have introduced a threat model named CIAA threat model. This model addresses all the safety problems particularly, Confidentiality, Integrity, convenience and Authentication. In inward at this model, authors have organized the threats on a distributed storage system underneath every class of the CIAA pillars of security and provided techniques that may be accustomed circumvent the threats. the opposite security model mentioned by the authors is that the knowledge Lifecycle Model that examines the kinds of threats that will extinction. underneath this model threats are organized underneath six teams and solutions are planned [22].

Dikaliotis, Dimakis and metal have planned a straightforward linear hashing technique that may observe errors within the storage nodes within the encoded distributed storage systems [23]. reciprocally Cooperative Recovery (MCR) mechanism permits the system to recover knowledge in things of multiple node failures. The transmission theme and style a linear network writing theme supported(n, k) strong-MDS code planned facilitate recover systems from failure with relative ease [14].

Hence it will be seen that the safety schemes within the distributed storage systems chiefly target knowledge security in terms of integrity and failure management (availability). 


\section{Distributed Database Security}

Distributed direction systems threats compared to face additional security their counterpart centralized information systems. the event of security for distributed information systems became additional sophisticate $\mathrm{d}$ with the introduction of many new information models comparable to electronic information service model, temporal information model, object computer database model etc.

In ancient security model, all information the info the information \} keep in information and therefore the users World Health Organization access that data belong to identical security level.

A structure secure information system assigns security level to ever group action and information. Clearance level of a group action is diagrammatical by security level assigned thereto and therefore the classification level of knowledge is given by the classification level. A structure secure direction system (MLS/DBMS) restricts information operations supported the protection levels [24]. From the higher than discussion, it are often seen that by introducing the military data classification and access management security of distributed databases are often increased.

Zubi has given a style that may improve the quantifiability, accessibility and adaptability whereas accessing varied forms of information during a distributed information system. He has conjointly projected multi level access management, confidentiality, responsibility, integrity and recovery to manage the protection of a distributed information system [25].

\section{CONCLUSION}

we have noted up to now, distributed systems are a vital a part of peoples' lives as a results of innovations within the recent past within the space of Web-based applications, and can still build a significant impact within the future. rising technologies equivalent to Grids can drive succeeding wave of innovation enabling the creation of applications that deliver IT because the fifth utility when water, electricity, gas, and also the phone phone. last, distributed computing may be a terribly broad space with immense potential to boost potency of business processes and quality of life!

From the higher than discussion, it are often seen that security becomes additional distinguished once the systems are distributed across over multiple geographic locations. every variety of distributed system has its own peculiar security necessities. But, all the systems have the common CIA triad because the heart of any security implementation. In computing clusters and grids the safety principally concentrates on protective the info in transit and access to distributed resources. Security in clusters is some what easier compared to grid because of homogenised nature of clusters. one in all the most attacks that has been allotted on clusters is that the Denial of Service (DoS) attack. Researchers have projected novel strategies supported Mark off chain to mitigate the impact of DoS attacks.
In grid the middleware layer provides the platform for the implementation of security on the whole grid system. Grid system user oust security supported PKI and X.509 certificates. The user authentication module within the grid provides security against threats by external sources and illegal actions by internal users.

Security of distributed storage systems principally focus on securing information. the most aras targeting distributed storage are protection against information corruption and protection of knowledge in things of node failures. Researchers have projected varied models and schemes to safeguard the storage system against attacks and node failures.

In distributed information system, the safety implementationhasbeen created additional difficult beca use of the supply of various sorts of information models. How ever researchers have shown that by applying multi level security supported military data classification and access management, distributed information security are often increased.

In this paper, the event of distributed systems was mentioned in terms of what a distributed system is and also the objectives of fixing a distributed system. From all the accessible distributed systems, four most typically used distributed systems were mentioned thorough and so the safety problems vis aged by these systems and also the solutions projected by varied researchers were mentioned thorough. Finally the safety problems and solutions projected for various systems were summarized and compared with one another.

\section{REFERENCES}

[1] G. Couloris, J. Dollimore and T. Kinberg, "Distributed Systems Concepts and Design", 4th Edition, Addison-Wesley, Pearson Education, UK, 2001.

[2] A. Tanenbaum and M. Van Steen, "Distributed Systems: Principles and Paradigms", Prentice Hall, Pearson Education, USA, 2002.

[3] R. Buyya (editor), "High Performance Cluster Computing", Prentice Hall, USA, 1999.

[4] I. Foster and C. Kesselman, "The Grid: Blueprint for a Future Computing Infrastructure", Morgan Kaufmann Publishers, USA, 1999.

[5] R. Subramanian and B. Goodman, "Peer-to-Peer Computing: Evolution of a Disruptive Technology", Idea Group, 2005.

[6] A. Luther, R. Buyya, R. Ranjan and S. Venugopal, "Peer-to-Peer Grid Computing and a NET-based Alchemi Framework", In High Performance Computing: Paradigm and Infrastructure, 2005.

[7] D.K. De Carvalho, P.R. Lyra and R.B. Willmersdorf, "A First Step towards a Petroleum Reservoir Simulator Using an Edge-Based Unstructured Finite Volume Formulation”, 2003.

[8] Ronald Scrofano, Maya B. Gokhale, Frans Trouw, and Viktor K. Prasanna, "Accelerating Molecular Dynamics Simulations with Reconfigurable Computers", IEEE Transactions on Parallel and Distributed Systems, Vol. 19, No. 6, Pp. 764-778, 2008.

[9] W. Sun, J. Shu, and W. Zheng, "Parallel Seismic Propagation Simulation in Anisotropic Media by Irregular Grids Finite Difference Method on PC Cluster", in Computational Science and Its Applications, Pp. 762-771, 2005.

[10] K. Zhang, P. Jun Dong, B. Ma, B. Yong Tang and H. Cai, "Innovation of IT Service in Textile Industrial Clusters from the Service System Perspective", in International Conference on Systems and Intelligent Management, Pp. 1819 - 1822, 2010.

[11] R. Buyya and S. Venugopal, "Market Oriented Computing and Global Grids: An Introduction", In Market Oriented Grid and Utility Computing, Rajkumar Buyya and Kris Bubendorfer, Vol. 1, Pp. 3-27, 2010.

[12] H. Ye, "Using Metadata Snapshots for Extending Ant-Based Resource 
Discovery Service in Inter-cooperative Grid Communities", In Proceedings of the First International Conference on Evolving Internet, Cap, Pp. 89-94, 2009.

[13] Y. Hu, Y. Xu, X. Wang, C. Zhan and P. Li, "Cooperative Recovery of Distributed Storage Systems from Multiple Losses with Network Coding", IEEE Journal on Selected Areas in Communications, Vol. 28, No. 2, Pp. 268-276, 2010.

[14] X. Gao Yu and W. Xing Li, "A new network storage architecture based on NAS and SAN", in 10th International Conference on Control, Automation, Robotics and Vision (ICARCV 2008), Pp. 2224-2227, 2008.

[15] A. Safari Mamaghani, M. Mahi, M. Reza Meybodi, and M. Hosseinzadeh Moghaddam, "A Novel Evolutionary Algorithm for Solving Static Data Allocation Problem in Distributed Database Systems", in Second International Conference on Network Applications, Protocols and Services, Pp. 14-19, 2010.

[16] T. Xie and X. Qin, "Security-Aware Resource Allocation for Real-Time Parallel Jobs on Homogeneous and Heterogeneous Clusters", IEEE Transactions on parallel and distributed Systems, Vol. 19, No. 5, Pp. 682-697, 2008.

[17] W. Li and Rayford B. Vaughn, "Cluster Security Research Involving the Modeling of Network Exploitations Using Exploitation Graphs", In Sixth IEEE International Symposium on Cluster Computing and the Grid Workshops, Pp. 26-36, 2006.

[18] Z. Jiang, S. Yan and L. Wang, "Survivability Evaluation of ClusterBased Wireless Sensor Network under DoS Attacks", In 5th International Conference on Wireless Communications, Pp. 1-4, 2009.

[19] Y. Demchenko, C. De Laat, O. Koeroo and D. Groep, "Re-thinking Grid Security Architecture", In IEEE Fourth International Conference on eScience, Pp. 79-86, 2008.

[20] Q. Xing, S. Xue and F. Liu, "Research of Grid Security Authentication Model", In International Conference on Computer Application and System Modeling (ICCASM), Vol. 1, Pp. 78-80, 2010.

[21] R. Hasan, S. Myagmar, Adam J. Lee and W. Yurcik, "Toward a threat model for storage systems", In Proceedings of the 2005 ACM Workshop on Storage Security and Survivability, 2005, Pp. 94-102.

[22] Theodoros K. Dikaliotis, Alexandros G. Dimakis and T. Ho, "Security in distributed storage systems by communicating a logarithmic number of bits", In IEEE International Symposium on Information Theory Proceedings, Pp. 1948 - 1952, 2010.

[23] N. Kaur, R. Singh, A.K. Sarje and M. Misra, "Performance evaluation of secure concurrency control algorithm for multilevel secure distributed database system", In International Conference on Information Technology: Coding and Computing (ITCC 2005), Vol. 2, No. 2, 2005, Pp. 249-254.

[24]Z. Suliman Zubi, "On distributed database security aspects", In International Conference on Multimedia Computing and Systems, Pp. 231-235, 2009. 